\title{
Teoría del Comportamiento Planificado e intención de infringir normas de transito en peatones. ${ }^{1}$
}

\author{
Emilio Moyano Díaz \\ Universidad de Santiago de Chile
}

\begin{abstract}
Resumo:
Desde la teoría del comportamiento planificado, se explora el comportamiento infractor de normas de tránsito en peatones y se prueba una escala de autoreporte acerca de cuán frecuentemente éstos infringen normas de tránsito, cometen errores y tienen lapsus al usar las vías. Participaran 146 habitantes de la ciudad de Santiago de Chile seleccionados intencionalmente, constituyendo un grupo balanceado a) por sexo, b) con y sin licencia para conducir, c) por edad (jóvenes de 17 a 25 años y adultos de 26 y más), y d) habiendo tenido y no alguna experiencia de accidente de tránsito, quienes completaron voluntaria y anónimamente un "Cuestionario de opinión sobre el comportamiento peatonal" y una "Escala de Comportamiento Peatonal". La actitud hacia la conducta, la norma subjetiva y el control percibido explican alrededor de un $40 \%$ de la varianza en la intención de realizar el comportamiento infractor ( $\mathrm{R}$ Múltiple= .6266; R2=.3927; F=30.609; Sig.F p<.0000). También se registra que el comportamiento peatonal difiere significativamente $(\mathrm{p}<.001)$ en las tres variables del modelo para los jóvenes vs. los adultos, mostrándose que los primeros tienen una actitud más positiva hacia el comportamiento infractor, perciben la norma subjetiva como menos inhibidora de la transgresión, aparecen con un menor control sobre el comportamiento infractor y con una intención conductual de mayor probabilidad a desarrollarlo. El resultado de la Escala de Comportamiento Peatonal muestra que los peatones jóvenes cometen más transgresiones, errores y lapsus al transitar que los adultos ( $\mathrm{p}<.001)$.
\end{abstract}

Palabras Clave: comportamiento planificado, peatones, infracción, tránsito. 
Key-words:

\begin{abstract}
Theory of Planned Behavior and pedestrians' intention to violate transit rules. Based on the Theory of Planned Behavior, the paper analyzes traffic rules violating behavior in pedestrians, testing a self-report scale that deals with frequency of transit norms violations, error committing and having lapses when using the streets. 146 inhabitants of the city of Santiago were intentionally selected, making up a balanced sample in terms of a) genre, b) having or not driving license, c) age (young, 17-25 years, or adult, 26 years or more), and d) with experience of transit accident or not, who voluntarily and anonymously completed a "Questionnaire of opinion about pedestrian behavior" and a "Scale of Pedestrian Behavior". The attitude towards behavior, the subjective norm and the perceived control explain about $40 \%$ of the variance of intention to transgress. Pedestrian behavior is also significantly different for young vs. adults for the three variables of the model, demonstrating that the first are more prone to violating behavior, perceive the subjective norm as less inhibiting of transgression, and exhibit less control over violating behavior with behavioral intention of higher probability to make it happen. The results of the Scale of Pedestrian Behavior indicate that young pedestrians perpetrate more transgressions, errors and lapses when in transit than adults.
\end{abstract}

\section{El problema}

$\mathrm{L}$ os accidentes de tránsito son tema de creciente interés en sociedades en desarrollo con altos niveles de crecimiento económico y, como consecuencia, de motorización. En Chile, a diferencia de los países con mayor seguridad de tránsito, un importante actor y provocador de accidentes de tránsito es el peatón; así, en este trabajo se explora el comportamiento del peatón en su transitar por la ciudad y, más particularmente, su conducta de riesgo y de infracción a las normas reguladoras de su comportamiento como usuario de vías públicas. 
Durante 1995 se registraron 50.790 accidentes de tránsito con un costo en vidas de alrededor de 1890 fallecidos por esta causa en Chile (Carabineros de Chile, 1996). Esta cifra debe ser corregida, incrementándosele, especialmente si se desea una comparación internacional, ya que la institución que hace el registro considera como 'muerto por accidente de tránsito' aquel al que la muerte le sobreviene solo hasta 24 horas después de ocurrido el accidente, tiempo que en la mayoría de los países europeos, por ejemplo, se prolonga hasta 3 o más meses después de ocurrido el evento. Los atropellos -junto con los choques- son el segundo tipo de accidentes más frecuente después de la colisión entre vehículos, sin embargo, es el tipo de accidente que más contribuye con casos fatales ( $45.8 \%$ del total).

En este contexto hemos desarrollado una línea de trabajo que explora actitudes hacia las transgresiones y las atribuciones causales que los conductores y los peatones construyen acerca de los accidentes de tránsito y que hemos constatado no corresponden a aquellas causas que más frecuentemente explicarían los accidentes según los expertos. Los habitantes de la ciudad tienden sistemáticamente a desaprobar el comportamiento transgresor, no presentando en general actitudes permisivas respecto del mismo (Moyano Díaz, 1997).

La teoría del comportamiento planificado (Ajzen, 1988, 1985) recientemente ha comenzado a aplicarse al estudio de las actitudes al conducir y los intentos por predecir el comportamiento infractor en conductores están dando sus primeros resultados (Parker, Manstead \& Stradling, 1995; Parker, Manstead, Stradling \& Reason, 1992a, 1992b). En el presente estudio se explora la aplicabilidad de esta teoría al comportamiento de cruzar las calles en zonas sin demarcación de protección para el peatón (a mitad de cuadra por ejemplo) toda vez que éste aparece como actor causal de una importante cantidad de accidentes de tránsito (alrededor de un $17.34 \%$ del total). Estudios previos realizados en conductores han subrayado la importancia de distinguir entre comportamiento transgresor en tanto violación (calculada o deliberada) de otro de un orden distinto que ha sido designado como error (Reason, Manstead, Stradling, Baxter \& Campbell, 1990). Esta conceptualización es transferida al comportamiento peatonal en el presente estudio. 
Se construyeron dos cuestionarios con los que se intenta, en el primero, evaluar las variables de la teoría del comportamiento planificado respecto del comportamiento de cruzar la calle en zonas desprotegidas o no demarcadas como cruce peatonal permitido. En éste, se evalúan la actitud hacia el comportamiento riesgoso, las normas subjetivas respecto al mismo, el control percibido y la intención conductual de realizar o no dicho comportamiento. Por otra parte, en el segundo instrumento, se sistematiza el autoreporte del comportamiento habitual de los peatones al transitar por la ciudad, intentándose diferenciar con él entre comportamientos de transgresión de aquellos otros tipificados como errores y de otros denominados lapsus. Los propósitos de este estudio son dos: 1.- Explorar la aplicabilidad de la teoría del comportamiento planificado para la explicación del comportamiento peatonal infractor y, 2.- Objetivar la frecuencia reportada de comportamientos peatonales tipificados como violaciones, errores y lapsus.

\section{Metodología}

\section{Muestra}

Se seleccionó intencionadamente una muestra balanceada de 146 participantes de la ciudad de Santiago, hombres y mujeres, jóvenes (17-25; $n=55)$ y adultos (26 y más; $n=91$ ), de los cuales 74 cuentan con licencia para conducir y 72 no disponen de ella (peatones). Del total de la muestra, la mitad ha participado alguna vez en algún accidente de tránsito en los últimos cinco años.

\section{Instrumentos}

Inspirado en un cuestionario inglés (Parker et al., 1992b) se construyó un cuestionario de opiniones sobre la conducta peatonal y de tránsito, constituído por 29 ítems tipo likert de 5 puntos relativos a una historia en la que el protagonista (el que responde) “... transitando a mitad de cuadra por una calle medianamente transitada en Santiago en día despejado, observa en la vereda de enfrente algo que necesita o llama su atención (negocio o persona conocida)... Ud. decide cruzar allí mismo la calle". Los items likert recogen la actitud hacia esa 
conducta transgresora, la norma subjetiva y el control percibido respecto a la misma (en Anexo I se encuentran ejemplos de ítems del instrumento). El segundo instrumento es una "Escala de Comportamiento Peatonal" con 26 items tipo likert de 6 puntos referidos a comportamientos transgresores o errores o lapsus al transitar como peatón por la ciudad; el respondente debe indicar para cada item de comportamiento cuán frecuentemente lo realiza, en una escala que va de $1=$ Nunca a $6=$ Siempre. Contiene 11 items relativos a transgresión (de comportamientos regulados por la ley de tránsito chilena), 7 al error, 4 a lapsus y 4 distractores (Anexo II).

\section{Procedimiento}

Un grupo de estudiantes universitarios de psicología fueron entrenados para aplicar el cuestionario a las muestras balanceadas, tal y como se indicó.

\section{Resultados}

En primer lugar se describen los resultados respecto del primer instrumento, evaluándose por medio de diferencias de medias (valor

Tabla 1. Promedios grupales para cada variable del modelo y significancia de las diferencias (t) $(\mathrm{N}=146)$.

\begin{tabular}{|c|c|c|c|c|c|c|c|c|}
\hline & \multicolumn{7}{|c|}{ Grupos } \\
\cline { 2 - 8 } & Joven & Adulto & Hombre & Mujer & Chofer & Peaton & Accid. & No-Acc \\
\hline $\begin{array}{c}\text { Actitud a } \\
\text { Conduta }\end{array}$ & 3.12 & $3.76^{* * *}$ & 3.22 & 3.48 & 3.5 & 3.22 & 3.22 & 3.53 \\
$\begin{array}{c}\text { Norma } \\
\text { Subjetiva } \\
\text { Control } \\
\text { Percebido } \\
\begin{array}{c}\text { Intención } \\
\text { Conductual }\end{array}\end{array}$ & 2.92 & $3.35^{\star * *}$ & 3.08 & 3.12 & 3.23 & 2.96 & 3.06 & 3.14 \\
\hline
\end{tabular}

1=Muy positivo 2=Bastante positivo 3=Algo Positivo 4=Poco Positivo 5= Nada Positivo. A menor valor, mayor actitud pro-transgresión, salvo para C.P.. Diferencias significativas t con $\mathrm{p}^{\text {wat }}$ $<.001 ;{ }^{* *} \mathrm{p}<0.05$ 
t) para cada grupo estudiado -por edad, sexo, status de chofer o peatón y de accidentado o no- respecto a cada una de las variables del modelo. En una segunda parte se analiza el valor predictivo de las variables Actitud hacia la conducta, Norma Subjetiva y Control Percibido sobre la Intención Conductual de realizar el comportamiento transgresor. Finalmente se da cuenta de los resultados obtenidos en la Escala de Comportamiento Peatonal.

Los promedios que difieren significativamente entre sí corresponden a la variable edad: los jóvenes de 17 a 25 años presentan promedios inferiores en tres de las cuatro variables del modelo y superior en una cuarta. Así, la actitud hacia el comportamiento transgresor de cruzar la calle por donde no se debe es más favorable en los jóvenes que en los adultos, la percepción que los jóvenes tienen acerca de cuáles son las creencias de los otros significativos respecto al comportamiento transgresor es de que más bien aprobarían la realización de dicho comportamiento; también a los jóvenes les costaría más abstenerse de realizar el comportamiento apropiado (control percibido) y su intención conductual es de que sería bastante probable que realizaran el comportamiento transgresor referido.

Explorando la adecuación del modelo del comportamiento planificado a este tipo de comportamientos peatonales, una regresión múltiple para el conjunto del modelo aparece explicando aproximadamente un $40 \%$ de la variabilidad de la intención conductual (F=30.60; Sig. F= .0000; Múltiple R= .6266; R Square= .3927). Las tres variables de la teoría del comportamiento planificado contribuyen significativamente a explicar las variaciones de la intención conductual (Sig T .0000, .005 y .0000, respectivamente para Actitud hacia la Conducta, Norma Subjetiva y Control Conductual Percibido) de realizar el comportamiento transgresor y/o riesgoso considerado como variable dependiente. La matriz de correlaciones (Pearson) entre las variables del modelo se presenta en la Tabla 2.

La matriz registra variables del modelo que están de moderada (.521) a escasamente (-.138) correlacionadas entre sí. La correlación más alta corresponde a la variable Actitud hacia la Conducta con la Intención Conductal y la más baja a la de Norma Subjetiva con Control 
Tabla 2. Matriz de correlaciones entre las variables del modelo

\begin{tabular}{|c|c|c|c|c|}
\hline $\begin{array}{c}\text { Intención } \\
\text { Conductual } \\
\text { Conductual }\end{array}$ & 1.000 & $\begin{array}{c}\text { Actitud a } \\
\text { la Conduta }\end{array}$ & $\begin{array}{c}\text { Norma } \\
\text { Subjetiva }\end{array}$ & $\begin{array}{c}\text { Control } \\
\text { Conductual }\end{array}$ \\
$\begin{array}{c}\text { Actitud a la } \\
\text { Conduta } \\
\text { Norma } \\
\text { Subjetiva } \\
\text { Control } \\
\text { Conductual }\end{array}$ & .521 & 1.000 & .356 & -.380 \\
\hline
\end{tabular}

Conductual. Desde un punto de vista gráfico se puede observar la Figura 1 que permite apreciar los resultados para el conjunto de relaciones del modelo.

Figura 1. Teoria del Comportamento Planificado aplicada a la intención de comportarse transgrediendo reglas em peatones $(\mathrm{N}=146)$

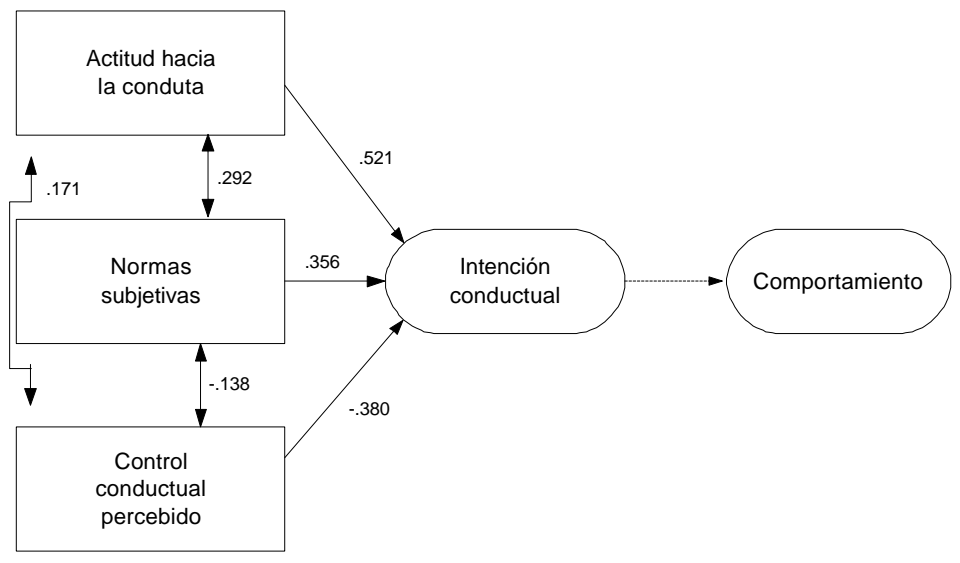

Se puede observar que las variables independientes están escasamente correlacionadas lo que contribuye a afirmar su independencia recíproca. De éstas la que aparece más altamente correlacionada con la variable dependiente comportamiento transgresor es la actitud hacia la conducta (.521) sugiriéndose así que 
el comportamiento peatonal transgresor estaría controlado más por la actitud que por la norma, no obstante la incidencia de ésta última es también importante.

En lo que respecta al reporte de comportamientos cotidianos que hemos tipificado como transgresiones, errores y lapsus al transitar por la ciudad, se registran algunas diferencias como se observa en Tabla 3.

Tabla 3. Promedios y significancia de "t" según condición de accidentado o no, conductor o no, etaria (17-25 y más) y de género $(n=146)$,

\begin{tabular}{|c|c|c|c|}
\hline \multirow[b]{2}{*}{ Grupos } & \multicolumn{3}{|c|}{ Comportamientos } \\
\hline & Transgressores & Erróneos & Lapsus \\
\hline Accidentes & 2.60 & 3.14 & 2.82 \\
\hline No Accidentes & 2.40 & 3.00 & 2.78 \\
\hline Conductor & 2.50 & 3.08 & 2.82 \\
\hline No Conductor & 2.52 & 3.08 & 2.77 \\
\hline Jóvenes & $2.74^{*}$ & $3.21^{*}$ & $3.05^{*}$ \\
\hline Adultos & $2.13^{*}$ & $2.87^{*}$ & $2.38^{*}$ \\
\hline Hombres & $2.77^{*}$ & 3.01 & 2.74 \\
\hline Mujeres & $2.29^{*}$ & 3.14 & 2.84 \\
\hline
\end{tabular}

1= Nunca, 2 = Casi Nunca, 3 = Ocasionalmente, 4= Frecuentemente, $5=$ Casi Siempre, $6=$ Siempre (A mayor valor, mayor frecuencia del comportamiento) * Diferencias significativas t con $p<.001$.

Los resultados muestran diferencias significativas según edad en la dirección de que los jóvenes reportan cometer un mayor número de comportamientos transgresores, erróneos y de lapsus al transitar por la ciudad, comparados con los adultos. Del mismo modo, respecto a la variable género se registra que los hombres reportan cometer más frecuentemente comportamientos transgresores que las mujeres, no diferenciándose en cambio respecto a errores ni en los lapsus al transitar, ya que personas de ambos géneros los realizan por igual (y corresponden a la categoría de 'frecuentemente').

Los resultados apoyan la idea de que los peatones contribuyen de modo importante a ser causa de y/o a verse involucrados en accidentes de tránsito en la medida en que parecen estar desplegando frecuentemente comportamientos transgresores (sancionados por la 
ley de tránsito), de alto riesgo y errores. De éstos, los que aparecen con un valor promedio indicativo de mayor frecuencia son los de "Desciendo de microbuses por el acceso" (puerta delantera), "Cruzo calles en zonas de cruces no protegidos" y "Cruzo por delante y por detrás de vehículos detenidos".

\section{Discusion y Conclusiones}

Algunas de las infracciones posibles de cometer por los peatones tipificadas en la Ley de Tránsito en Chile (1996, №18.290) son "Peatón en la calzada"; "Cruzar repentinamente la calzada; "Imprudencia de los peatones"; "Transitar en estado de ebriedad", las tres primeras de las cuales hemos resumido en la situación sometida a análisis mediante la aplicación del modelo de comportamiento planificado. La exploración de distintas variables de los participantes -condición de haber sufrido accidente o no, chofer o peatón, edad y sexo- muestra que solo estas dos últimas introducen diferencias. Los peatones jóvenes de 17 a 25 años aparecen consistentemente estando más a favor de la realización de la conducta infractora que los adultos, en su actititud, norma subjetiva y control conductual percibido. La intención conductual de realizar la infracción es significativamente mayor en ellos que en los adultos. Muy consistentemente con esto, los peatones jóvenes declaran cometer un mayor número de infracciones, de errores y de lapsus al transitar por la ciudad, cuando se les compara con los adultos. Los hombres reconocen también más errores que las mujeres al transitar, no diferenciándose en cambio respecto de errores ni lapsus al usar las vias. Estos resultados van en la dirección de lo mostrado por la literatura internacional relativa al comportamiento de conductores; las personas mayores reportan menor intención de cometer infracciones y los hombres asumen mayor comportamiento de riesgo que las mujeres al conducir (Parker, Manstead, Stradling, Reason y Baxter, 1992; Parker Manstead y Stradling, 1995). Pues bien, como peatones se observa un comportamiento en la misma dirección.

El modelo de comportamiento planificado parece adecuado para la predicción de las intenciones conductuales de los peatones ya que 
explica una importante porcentaje de la varianza del mismo. Al no haber, en nuestro conocimiento, experiencias previas de este tipo de aplicaciones (al comportamiento peatonal), consideramos que su uso a estos efectos resulta muy promisorio. El modelo permite objetivar que los peatones manifiestan una intención conductual que refleja claramente una decisión (consciente por tanto) de infringir la norma, es decir, se trata en general de usuarios de las vias quienes deliberadamente despliegan un comportamiento riesgoso. El que su comportamiento infractor aparezca actitudinalmente más que normativamente determinado es coherente con el hecho de que en nuestra sociedad no está para nada incorporado que el peatón pudiese ser multado por su comportamiento infractor como usuario de las vias. ¿No ha llegado el momento de comenzar a controlar el comportamiento peatonal en nuestras ciudades para evitar más muertes y juicios caros, lentos y generalmente infructuosos? ¿No es acaso la hora ya de "castigar" económicamente al peatón como se hace de antaño con el conductor cuando infringe normas como usuario de las vias en el espacio público?

Por otra parte, los resultados encontrados sugieren recomendar a nivel de campañas de prevención y de educación de tránsito el corregir la percepción, bastante extendida en el medio nacional, de que el peatón sería una 'víctima' de choferes agresivos e inescrupulosos y más bien comenzar a idear campañas de prevención relativas a generar en aquellos una actitud de mayor conformidad con las normas peatonales de uso de vias. Es muy probable que muchos peatones desconozcan cuáles son los comportamientos propios o adecuados a su rol y esa información debería estar incorporada tanto en la educación regular de tránsito en los colegios, así como en campañas por los medios masivos de comunicación. Es razonable pensar sin embargo, que la práctica cotidiana de observar a modelos adultos de comportamiento peatonal inadecuado pese fuertemente en la socialización de los niños ya que es habitual observar en las calles -especialmente en sectores céntricos populares (Estación Central, por ejemplo) que familias completas atraviesen grandes avenidas en medio de cuadras donde es inexistente el paso peatonal regulado. Para ellos normalmente no hay reconvención -ni menos multa- de parte de la autoridad de tránsito 
por su comportamiento. El comportamiento transgresor o riesgoso en tránsito es altamente resistente a la extinción pues es normalmente recompensado o, al menos, está libre de consecuencias negativas. Las personas que atraviesan donde no se debe con sus hijos pequeños "a la rastra" o en brazos alcanzan su objetivo generalmente sonrientes. Cuando eventualmente se enojan es porque alguno de sus hijos titubeó en la partida o inicio del comportamiento o durante su ejecución. La educación para algunos y el recordatorio para otros, acerca de cuáles son los comportamientos peatonales adecuados en las ciudades podrían ser exigidos, por ejemplo en cartillas ubicables en los asientos en el transporte interprovincial, al igual que aquellas que existen en el transporte aéreo, donde además se enseña y recuerda a los pasajeros, toda y cada vez que se suben a un avión, cuáles son las medidas de seguridad pertinentes. Construir seguridad de tránsito es mejorar la calidad de vida.

\section{Referencias}

Ajzen, I. (1988). Attitudes, personality and behavior. Milton Keynes: Open University Press.

Ajzen, I. 1985. From intentions to actions: A theory of Planned Behavior. In J.Kuhl y J.Beckmann (Eds), Action Control: From cognition to behavior (pp. 11-38). Berlin: Springer-Verlag.

Carabineros de Chile (1995). Evaluación de los accidentes de tránsito del año 1995 a nivel nacional comparados con 1994. Santiago, Chile: Dirección de Fronteras y Servicios Especiales, Departamento de Servicios de Tránsito.

Moyano Díaz, E. (1997). Evaluation of traffic violation behaviors and the causal attribution of accidents in Chile. Environment and Behavior, 29 (2), 264-282.

Parker D., Manstead, A. S. R., Stradling, S. G. (1995). Extending the theory of planned behaviour: the role of personal norm. British Journal of Social Psychology, 34(2), 127-137.

Parker, D., Manstead, A. S., Stradling, S. G., \& Reason J. T. (1992a). Determinants of intention to commit driving violations. Accident Analysis and Prevention, 24(2), 117-131.

Parker, D., Manstead, A. S., Stradling, S. G., \& Reason, J. T. (1992b). Intention to commit driving violations: an application of the Theory of Planned Behavior. Journal of Applied Psychology, 77(1), 94101. 
346 Dossiê Psicologia Ambiental

Reason, D., Manstead, A. S., Stradling, S. G., Baxter, B., \& Campbell, K. (1990). Errors and violations on the roads: a real distinction? Ergonomics, 33, 1315-1332.

Anexo I: Cuestionario de opiniones sobre comportamiento vial (muestra)

Emilio Moyano Díaz

Escuela de Psicología,USACH.

En este cuestionario se describen diversas formas en que la gente se comporta en situaciones relacionadas con el tránsito. En cada situación usted deberá indicar cual sería su forma habitual de pensar o actuar. Las respuestas a este cuestionario son anónimas y se utilizarán sólo con fines de investigación; le rogamos que responda sinceramente. Para responder a este cuestionario elija solamente UNA de las cinco opciones ofrecidas para cada pregunta. Encierre en un círculo el número de la opción elegida, por ejemplo:

Me gusta caminar de noche

$\begin{array}{lll}\text { 1.Totalmente de acuerdo } & \text { 2. Bastante de acuerdo } & \text { 3.Algo de acuerdo } \\ \text { 4. Poco de acuerdo } & \text { 5. Nada de acuerdo. }\end{array}$

No deje ninguna pregunta sin contestar.

\section{Primera parte - Comportamiento Peatonal.}

Ud. camina por una calle medianamente transitada de Santiago, es un dia despejado y va a mitad de cuadra, relativamente apurado, cuando observa en la vereda de enfrente algo que Ud. necesita o que le llama la atención (negocio o persona conocida). Ud. decide cruzar allí mismo la calle.

a. Cruzar la calle me parecería..
1. Muy positivo
2. Bastante positivo
3. Algo positivo
4. Poco positivo
5. Nada positivo
Algo positivo

b. Cruzar la calle me evitaría muchas molestias.
1. Totalmente de acuerdo
2. Bastante de acuerdo
3. Algo de acuerdo
4. Poco de acuerdo
5. Nada de acuerdo

c. Cruzar la calle me expone a un mayor riesgo de accidente.
1. Totalmente de acuerdo
2. Bastante de acuerdo
3. Algo de acuerdo
4. Poco de acuerdo
5. Nada de acuerdo

d. Cruzar la calle podría significar ser multado.
1. Totalmente de acuerdo
2. Bastante de acuerdo
3. Algo de acuerdo
4. Poco de acuerdo
5. Nada de acuerdo

e. Llegar al lugar de la vereda de en frente que me propuse es algo que me importaría... 

1. Mucho
2. Bastante
3. Algo
4. Poco
5. Nada

etc.

Anexo II: Escala de Comportamiento Peatonal (muestra)

Emilio Moyano Díaz

Escuela de Psicología, USACH.

\section{Segunda parte- Comportamiento Peatonal}

Al transitar por la ciudad todos cometemos errores lapsus o transgresiones. Le rogamos que a cada item responda si se trata de una conducta que Ud. realiza Nunca (1), Casi Nunca (2), Ocasionalmente (3), Frecuentemente (4), Casi siempre (5) o Siempre (6).

\begin{tabular}{|c|c|c|c|c|c|c|}
\hline \multirow{2}{*}{$\begin{array}{l}\text { ¿Cuán a menudo Ud. hace las conductas que } \\
\text { aparecen a continuación? }\end{array}$} & \multicolumn{4}{|c|}{ Nunca } & \multicolumn{2}{|c|}{ Siempre } \\
\hline & 1 & 2 & 3 & 4 & 5 & 6 \\
\hline \multicolumn{7}{|l|}{$\begin{array}{l}\text { Cruzo la calzada (calle) cuando la luz verde está } \\
\text { intermitente cambiando al rojo. }\end{array}$} \\
\hline \multicolumn{7}{|l|}{ Olvido transitar por la derecha en las veredas. } \\
\hline \multicolumn{7}{|l|}{$\begin{array}{l}\text { Soy impaciente con el peatón de delante mío cuando } \\
\text { no atraviesa la calle cuando todavía puede y lo } \\
\text { adelanto. }\end{array}$} \\
\hline \multicolumn{7}{|l|}{$\begin{array}{l}\text { Me ubico por igual al costado derecho que al izquierdo } \\
\text { en las escaleras mecánicas. }\end{array}$} \\
\hline \multicolumn{7}{|l|}{$\begin{array}{l}\text { Espero que el semáforo cambie a luz verde parado } \\
\text { sobre la calzada y no sobre la vereda. Cruzo calles } \\
\text { pasando por delante y por detrás de vehículos } \\
\text { estacionados. }\end{array}$} \\
\hline \multicolumn{7}{|l|}{ Cruzo semáforos aún cuando no estén con luz verde } \\
\hline \multicolumn{7}{|l|}{$\begin{array}{l}\text { Cruzo calles en zonas de cruces no protegidos para } \\
\text { peatones. }\end{array}$} \\
\hline \multicolumn{7}{|l|}{$\begin{array}{l}\text { En sectores céntricos o concurridos de la ciudad cruzo } \\
\text { calles a mitad de cuadra. }\end{array}$} \\
\hline \multicolumn{7}{|l|}{$\begin{array}{l}\text { De repente me doy cuenta que he recorrido varias } \\
\text { calles y cruces sin prestar atención al tránsito. }\end{array}$} \\
\hline \multicolumn{7}{|l|}{$\begin{array}{l}\text { Miro la luz del semáforo de los automovilistas } \\
\text { adelantándome a cruzar cuando a ellos recién le están } \\
\text { cambiando a roja. }\end{array}$} \\
\hline \multicolumn{7}{|l|}{$\begin{array}{l}\text { Muy temprano en la mañana o muy tarde en la noche } \\
\text { desciendo a caminar por la calzada sin importar si } \\
\text { voy en la misma dirección que los vehículos. }\end{array}$} \\
\hline \multicolumn{7}{|l|}{$\begin{array}{l}\text { Hago el camino más corto para llegar al punto de la } \\
\text { vereda que quiero aún cuando esto significa saltarme } \\
\text { alguna barrera o hacer algo riesgoso. }\end{array}$} \\
\hline \multicolumn{7}{|l|}{ Desciendo o asciendo a micros en movimiento. } \\
\hline Etc. & & & & & & \\
\hline
\end{tabular}


348 Dossiê Psicologia Ambiental

\begin{tabular}{l|l} 
Nota & $\begin{array}{l}\text { Investigación realizada gracias al finan- } \\
\text { ciamiento otorgado por el Departamento }\end{array}$ \\
& de Investigaciones Científicas y Tecno- \\
& $\begin{array}{l}\text { lógicas (DICYT) de Universidad de San- } \\
\text { tiago de Chile. }\end{array}$
\end{tabular}

Emilio Moyano-Díaz doutorou-se em Psicologia pela Universidade Católica de Louvain, Bélgica e é Professor Titular e Subdiretor da Escola de Psicologia da Universidade de Santiago do Chile. Realiza pesquisas sobre satisfação residencial e clima social, percepção de riscos urbanos e acidentes de trânsito.

Endereço para correspondência: Av. Ecuador 3650, 3 piso, Universidad de Santiago de Chile, Santiago, Chile; Telefax (56)(2) (7761986).

E-Mail: emoyano@lauca.usach.cl 